\title{
Investigating the effects of the COVID-19 pandemic on the reasons for application to the emergency department in patients with neurological symptoms
}

\author{
Cemile Haki $^{1}$ D $\cdot$ Halil Kaya $^{2}$ D
}

Received: 23 December 2020 / Accepted: 5 April 2021 / Published online: 21 April 2021

(c) Belgian Neurological Society 2021

\begin{abstract}
Objective To investigate whether the COVID-19 pandemic had an effect on the emergency department admission complaints of patients with neurological symptoms.

Methods A total of 976 patients admitted to the emergency department of our hospital and had undergone neurology consultation during a 6-month period were evaluated. The reasons for consultation, the number of patients consulted, hospitalization counts, and imaging studies for neurological assessment including computerized tomography (CT) and magnetic resonance imaging (MRI), were recorded and compared.

Results Compared to the pre-pandemic period, there were significant decreases in the number of neurological consultations requested by the emergency department (overall and related to stroke, seizure and other reasons) and the number of patients hospitalized in the neurology department. We also found that the number of orders for cranial CT and MRI images during the pandemic period had decreased significantly.

Conclusion Restrictions, social isolation measures and patients' reluctance to apply to hospitals to avoid contact with possibly infected people may have led to a decrease in the number of patients with neurological symptoms admitted to the emergency department and the number of hospitalized patients.
\end{abstract}

Keywords COVID-19 pandemic $\cdot$ Emergency room $\cdot$ Neurological admission

\section{Introduction}

In December 2019, the first severe acute respiratory syndrome coronavirus 2 (SARS-CoV-2) disease (COVID-19) case was identified with the presence of pneumonia cases in a group of cases in Wuhan, China [1,2].

On January 30, 2020, the World Health Organization declared that the SARS-CoV-2 epidemic constituted a Public Health Emergency of International Concern, because the epidemic was caused by a coronavirus which was highly

Cemile Haki

cemilehaki@gmail.com

1 Department of Neurology, Bursa City Hospital, Doğanköy Neighb., Gümüş Ave \#10, 16110 Nilüfer, Bursa, Turkey

2 University of Health Sciences, Department of Emergency Medicine, Bursa Yuksek Ihtisas Training and Research Hospital, Bursa, Turkey homologous to SARS-CoV and MERS-CoV, two coronaviruses that had afflicted humans [3-5].

The SARS-CoV-2 virus, which affects many systems and organs besides the lung, presents with a wide range of clinical pictures, from asymptomatic infection to severe pneumonia with high rates of multi-organ failure and mortality. In various studies in the literature, it has been reported that COVID-19 affects the central nervous system (CNS) and peripheral nervous system (PNS), and may progress with neurological symptoms and complications [6, 7].

After the determination of the first cases in Turkey, on March 11, 2020, various restrictions and nationwide curfew were imposed upon the population to reduce disease spread and schools were closed on the 12th of March. Shortly after, on April 04, 2020, a targeted curfew was imposed upon people aged under than 20 years, completely restricting them from leaving their homes [8].

In studies conducted in Turkey, the frequency of neurological consultations among patients admitted to the emergency department has been reported in the range of 
0.83 to $6.9 \%$. In these studies, it was observed that the most common diagnosis after neurological evaluation was stroke (ischemic, hemorrhagic and transient ischemic attack) with a frequency of $24.7-70.6 \%$ [9, 10].

It was reported that patients were less likely to apply to hospital emergency departments during the COVID-19 pandemic [11]. During the pandemic period, it is expected to observe a decrease in the number of non-serious cases and related consultations in emergency departments. The important issue is the question of whether there is a decrease in the number of patients with neurological diseases that can be fatal and require rapid treatment, such as stroke. In various studies, it has been reported that the number of cases with severe neurological disease (such as acute stroke) decreased after the pandemic, and there were delays in the application/ admission of patients with acute stroke [12-15].

In our study, we conducted a comparison between the COVID-19 era and the pre-pandemic period in terms of the profiles and characteristics of patients who had undergone neurological consultation after being admitted to the emergency department, including demographic characteristics, reasons for their admission to the emergency department and the diagnoses they received after neurological evaluation. Subsequently, we aimed to investigate whether the pandemic had an effect on patients with neurological symptoms who were admitted to the emergency department, and the nature of these effects, if any.

\section{Materials and methods}

Our study was conducted as a retrospective cross-sectional study. This study conducted in a tertiary hospital which is located in the northwest of Turkey and 4th largest city in the country was held in Bursa City Hospital. It included patients presenting to the Adult Emergency Department of Bursa City Hospital (1355 beds). According to the data of 2020, the population catchment area of hospital's ER (Nilufer district) is 484.832 . Of them $240.819(49.67 \%)$ are men and $244.013(50.33 \%)$ are women. Among the 106,956 patients admitted to the emergency department of our hospital during the 6-month study period (pre-pandemic: 15 December 2019 to 14 March 2020, pandemic: 15 March 2020 to 15 June 2020), those who had undergone neurological consultation $(n=976)$ were included. Approval from Bursa City Hospital Ethics Committee and permission from the Ministry of Health and Bursa City Hospital administration were obtained for the use of patient data. The study was carried out in accordance with the principles of the Declaration of Helsinki. All data pertaining to the study were obtained from electronic hospital records.

Demographic characteristics, comorbid diseases, reasons for emergency consultation, diagnosis after neurological evaluation, radiological images [computerized tomography (CT), magnetic resonance imaging (MRI)], discharge status of the patients from the emergency department (discharge, hospitalization, referral to another hospital, refusal of treatment) and the latest status of the hospitalized patients (discharge, exitus) were recorded from the files of the patients.

A comparison was made between patients according to their date of application, in two groups: those that applied before the pandemic (BP) and those that applied after the first case of COVID-19 in Turkey (AP). Treatment refusal was defined as patients who were offered hospitalization but did not want to remain in the hospital for treatment. The complaints of the patients were evaluated under three main headings: cerebrovascular diseases, seizures and others.

Exclusion criteria: being younger than 18 years old and not having undergone neurological consultation.

\section{Statistical analysis}

All analyses were performed on SPSS v21 (SPSS Inc., Chicago, IL, USA). For the normality check, the Kolmogorov-Smirnov test was used. Data are given as mean \pm standard deviation or median (1st quartile-3rd quartile) for continuous variables according to normality of distribution, and as frequency (percentage) for categorical variables. Frequencies of the variables in the BP and AP periods were analyzed with one-sample chi-square tests with equal probabilities. Between-group comparisons of the continuous variables were performed with the independent samples $t$-test or the Mann Whitney $U$ test, depending on normality of distribution. Between-group comparisons of the categorical variables were performed with Chi-square or Fisher's exact tests. Statistical significance value was accepted as $p<0.05$.

\section{Results}

A total of 60,527 patients in the 3-month period before the pandemic and a total of 46,429 patients in the 3-month period during the pandemic had been evaluated in the emergency department. Neurology consultation was requested from 584 patients in the BP period and 392 patients in the AP period. Mean age was $64.88 \pm 17.09$ (range 18-104) years. The number of patients in the BP group was significantly higher than the number of patients in the AP group $(p<0.001)$. In addition, the number of patients discharged from the emergency department $(p<0.001)$, the number of hospitalizations in the neurology ward $(p<0.001)$, and the frequencies of stroke $(p=0.004)$, ischemic stroke $(p=0.014)$, seizure $(p=0.008)$ and other neurologic problems $(p<0.001)$ were significantly higher in the BP compared to the AP group. There were no significant differences 
between the BP and AP groups with regard to the number of patients hospitalized in the neurology intensive care unit (ICU), number treatment refusals, mortality in the neurology department, and the frequencies of hemorrhagic stroke and transient ischemic attack (TIA) (Table 1, Figs. 1, 2, 3).

When we evaluated all patients, there were no significant differences between the BP and AP groups with regard to age; there were 294 (50.34\%) males and 290 (49.66\%) females who applied before the pandemic, while 208 (53.06\%) males and 184 (46.94\%) females had applied after the pandemic $(p=0.405)$. The frequencies of hypertension $(p=0.025)$, cerebrovascular accident (CVA) history $(p=0.031)$, or impaired consciousness $(p=0.033)$ were significantly higher in the $\mathrm{BP}$ group compared to the AP group. Loss of strength $(p<0.001)$, visual impairment $(p=0.012)$ and paresthesia $(p=0.020)$, malignancy history $(p=0.039)$
Table 1 Comparison of the $\mathrm{BP}$ and AP groups in terms of imaging studies and clinical characteristics
Fig. 1 Status after emergency department before and after pandemic

\begin{tabular}{lrrrr}
\hline & BP $(n)$ & AP $(n)$ & Total $(n)$ & \multicolumn{1}{c}{$p$} \\
\hline Patient number $(n)$ & $584(59.84 \%)$ & $392(40.16 \%)$ & $976(100.00 \%)$ & $<\mathbf{0 . 0 0 1}$ \\
Computerized tomography & $567(59.50 \%)$ & $386(40.50 \%)$ & $953(100.00 \%)$ & $<\mathbf{0 . 0 0 1}$ \\
Magnetic resonance imaging & $451(58.95 \%)$ & $314(41.05 \%)$ & $765(100.00 \%)$ & $<\mathbf{0 . 0 0 1}$ \\
Status after emergency department & & & & \\
Discharged & $223(63.17 \%)$ & $130(36.83 \%)$ & $353(100.00 \%)$ & $<\mathbf{0 . 0 0 1}$ \\
Neurology clinic & $210(61.05 \%)$ & $134(38.95 \%)$ & $344(100.00 \%)$ & $<\mathbf{0 . 0 0 1}$ \\
Neurology intensive care unit & $56(51.85 \%)$ & $52(48.15 \%)$ & $108(100.00 \%)$ & 0.700 \\
Other clinic & $40(57.97 \%)$ & $29(42.03 \%)$ & $69(100.00 \%)$ & 0.185 \\
Other intensive care unit & $25(52.08 \%)$ & $23(47.92 \%)$ & $48(100.00 \%)$ & 0.773 \\
Refused treatment & $11(42.31 \%)$ & $15(57.69 \%)$ & $26(100.00 \%)$ & 0.433 \\
Referred another hospital & $19(67.86 \%)$ & $9(32.14 \%)$ & $28(100.00 \%)$ & 0.059 \\
Exitus & $20(51.28 \%)$ & $19(48.72 \%)$ & $39(100.00 \%)$ & 0.873 \\
Diagnosis & & & & \\
Stroke & $290(56.31 \%)$ & $225(43.69 \%)$ & $515(100.00 \%)$ & $\mathbf{0 . 0 0 4}$ \\
Ischemic & $206(56.44 \%)$ & $159(43.56 \%)$ & $365(100.00 \%)$ & $\mathbf{0 . 0 1 4}$ \\
Hemorrhagic & $25(58.14 \%)$ & $18(41.86 \%)$ & $43(100.00 \%)$ & 0.286 \\
Transient ischemic attack & $59(55.14 \%)$ & $48(44.86 \%)$ & $107(100.00 \%)$ & 0.288 \\
Seizure & $53(64.63 \%)$ & $29(35.37 \%)$ & $82(100.00 \%)$ & $\mathbf{0 . 0 0 8}$ \\
Other & $245(63.64 \%)$ & $140(36.36 \%)$ & $385(100.00 \%)$ & $<\mathbf{0 . 0 0 1}$ \\
\hline
\end{tabular}

Data are given as frequency (percentage)

$p$ values were obtained by one-sample chi-square test with equal probabilities

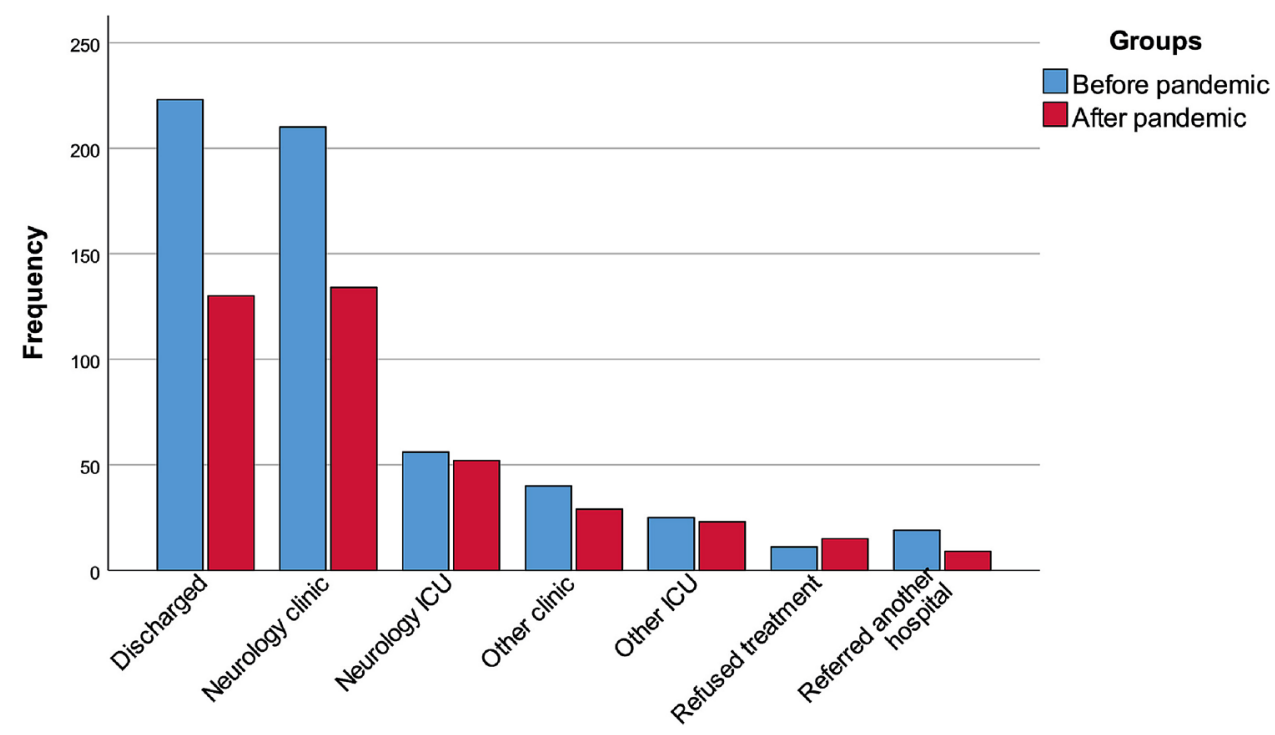

Status after emergency department 
Fig. 2 Stroke types of the patients before and after pandemic

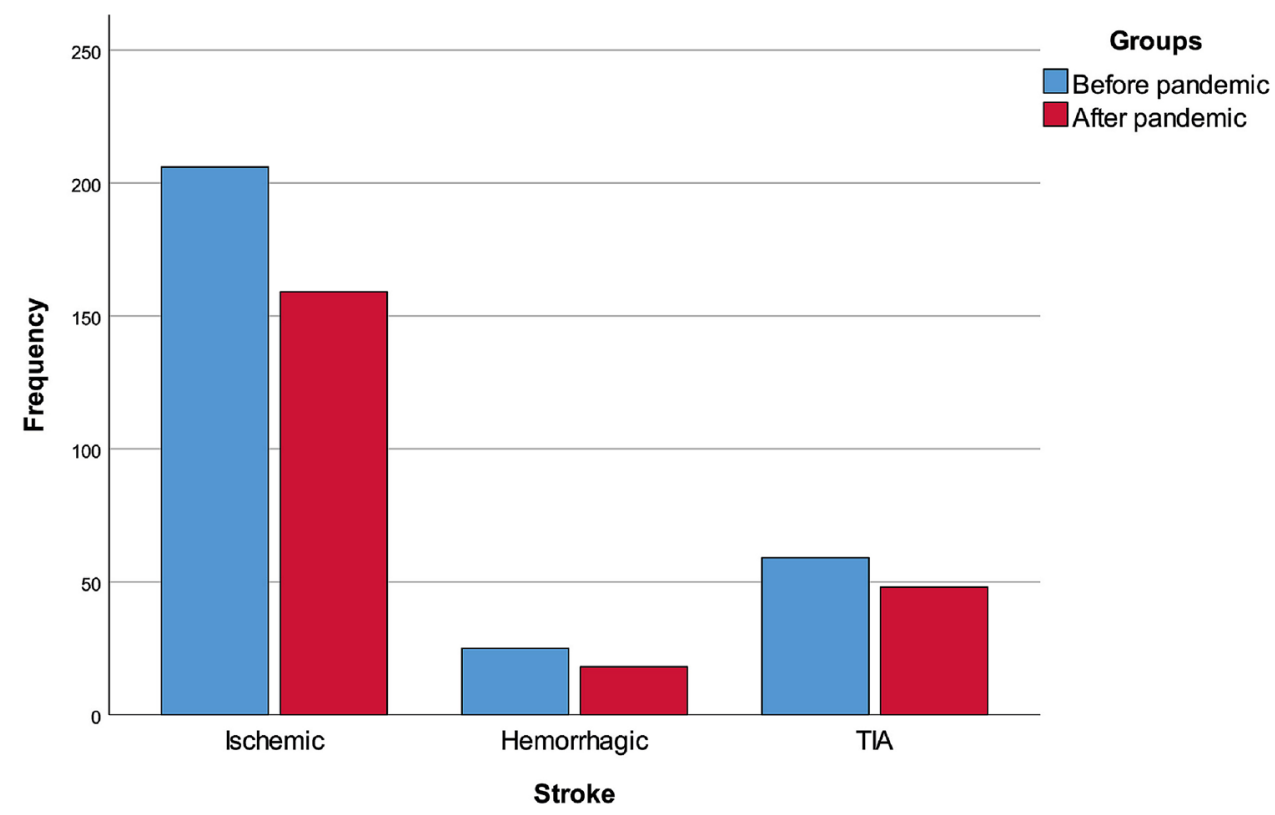

were significantly more common in the AP group than in the BP group. There were no significant differences between groups with regard to length of stay in hospital (Table 2).

When we evaluated patients with stroke, we found that age was significantly higher in the BP group than in the AP group $(p=0.014)$. The percentage of patients hospitalized in the neurology ward was significantly higher in the $\mathrm{BP}(p=0.016)$ group. Compared to the AP group, hypertension was significantly more common in the BP group $(p<0.001)$. There were no significant differences between the two groups with regard to CT, MRI, large vessel, small vessel, anterior system, posterior system stroke, exitus, disease history (except hypertension) (Table 3).

When we evaluated patients who had seizures, there were no significant differences between BP and AP with regard to age, gender and length of stay in hospital. Thirtytwo $(62.75 \%)$ patients had had their first seizure before the pandemic while $21(75.00 \%)$ patients had their first seizure after the pandemic $(p=0.391)$. Twelve $(22.64 \%)$ patients had status in the BP group, while $3(10.34 \%)$ cases of status
Table 2 Summary of admission characteristics in patients admitted before and after the pandemic

\begin{tabular}{lllr}
\hline & BP $(n=584)$ & AP $(n=392)$ & $p$ \\
\hline Age & $65.62 \pm 17.59$ & $63.78 \pm 16.27$ & 0.100 \\
Gender & & & \\
Male & $294(50.34 \%)$ & $208(53.06 \%)$ & 0.405 \\
Female & $290(49.66 \%)$ & $184(46.94 \%)$ & \\
Length of stay in hospital/day & $6(4-10)$ & $5(4-11)$ & 0.356 \\
Malignancy & $12(2.05 \%)$ & $18(4.59 \%)$ & $\mathbf{0 . 0 3 9}$ \\
Cerebrovascular accident history & $108(18.49 \%)$ & $52(13.27 \%)$ & $\mathbf{0 . 0 3 1}$ \\
Cause of admission to the hospital & & & \\
Impaired consciousness & $131(22.43 \%)$ & $66(16.84 \%)$ & $\mathbf{0 . 0 3 3}$ \\
Headache & $27(4.62 \%)$ & $15(3.83 \%)$ & 0.660 \\
Dizziness & $52(8.90 \%)$ & $24(6.12 \%)$ & 0.112 \\
Speech disorder & $75(12.84 \%)$ & $47(11.99 \%)$ & 0.693 \\
Loss of strength & $130(22.26 \%)$ & $128(32.65 \%)$ & $\mathbf{0 . 0 0 1}$ \\
Visual impairment & $6(1.03 \%)$ & $14(3.57 \%)$ & $\mathbf{0 . 0 1 2}$ \\
Syncope & $37(6.34 \%)$ & $19(4.85 \%)$ & 0.401 \\
Paresthesia & $37(6.34 \%)$ & $41(10.46 \%)$ & $\mathbf{0 . 0 2 0}$ \\
Cranial neuropathy & $22(3.77 \%)$ & $18(4.59 \%)$ & 0.637 \\
\hline
\end{tabular}

Data are given as mean \pm standard deviation or median (1st quartile-3rd quartile) for continuous variables according to normality of distribution and as frequency (percentage) for categorical variables 
Table 3 Summary of stroke patients' characteristics with regard to admission before and after pandemic

\begin{tabular}{llll}
\hline & BP $(n=290)$ & AP $(n=225)$ & $p$ \\
\hline Age & $69.73 \pm 13.19$ & $66.88 \pm 12.60$ & $\mathbf{0 . 0 1 4}$ \\
Gender & & & \\
Male & $147(50.69 \%)$ & $121(53.78 \%)$ & 0.487 \\
Female & $143(49.31 \%)$ & $104(46.22 \%)$ & \\
Length of stay in hospital/day & $5(1-8)$ & $4(0-7)$ & $\mathbf{0 . 0 0 9}$ \\
Hospitalized in neurology department & $236(81.38 \%)$ & $163(72.44 \%)$ & $\mathbf{0 . 0 1 6}$ \\
Exitus & $18(7.63 \%)$ & $18(11.04 \%)$ & 0.321 \\
Stroke type & & & \\
Ischemic & $206(71.03 \%)$ & $159(70.67 \%)$ & 0.942 \\
Hemorrhagic & $25(8.62 \%)$ & $18(8.00 \%)$ & \\
Transient ischemic attack & $59(20.34 \%)$ & $48(21.33 \%)$ & \\
Vessel & & & \\
Large & $38(18.81 \%)$ & $28(18.06 \%)$ & 0.857 \\
Small & $164(81.19 \%)$ & $127(81.94 \%)$ & \\
System & & & \\
Anterior & $162(80.20 \%)$ & $132(85.16 \%)$ & 0.280 \\
Posterior & $40(19.80 \%)$ & $23(14.84 \%)$ & \\
\hline
\end{tabular}

Data are given as mean \pm standard deviation or median (1st quartile-3rd quartile) for continuous variables according to normality of distribution and as frequency (percentage) for categorical variables were identified in the AP group $(p=0.281)$. There were no significant differences between groups with regard to CT, MRI, status after emergency department discharge, hospitalization in the neurology department, exitus, and disease history.

When we evaluated patients with other neurologic problems (patients without stroke or seizure), there were no significant differences between the BP and AP groups with regard to age, gender and length of stay in hospital (Fig. 3).

\section{Discussion}

In our study, it was determined that neurology consultation was requested by the emergency department for 584 patients in the pre-pandemic period and for 392 patients during the pandemic period. The frequency of overall consultation requests and consultations for stroke, seizure and other reasons had decreased in similar fashion to the number of patients hospitalized in the neurology department and
Fig. 3 Diagnosis of the patients before and after pandemic

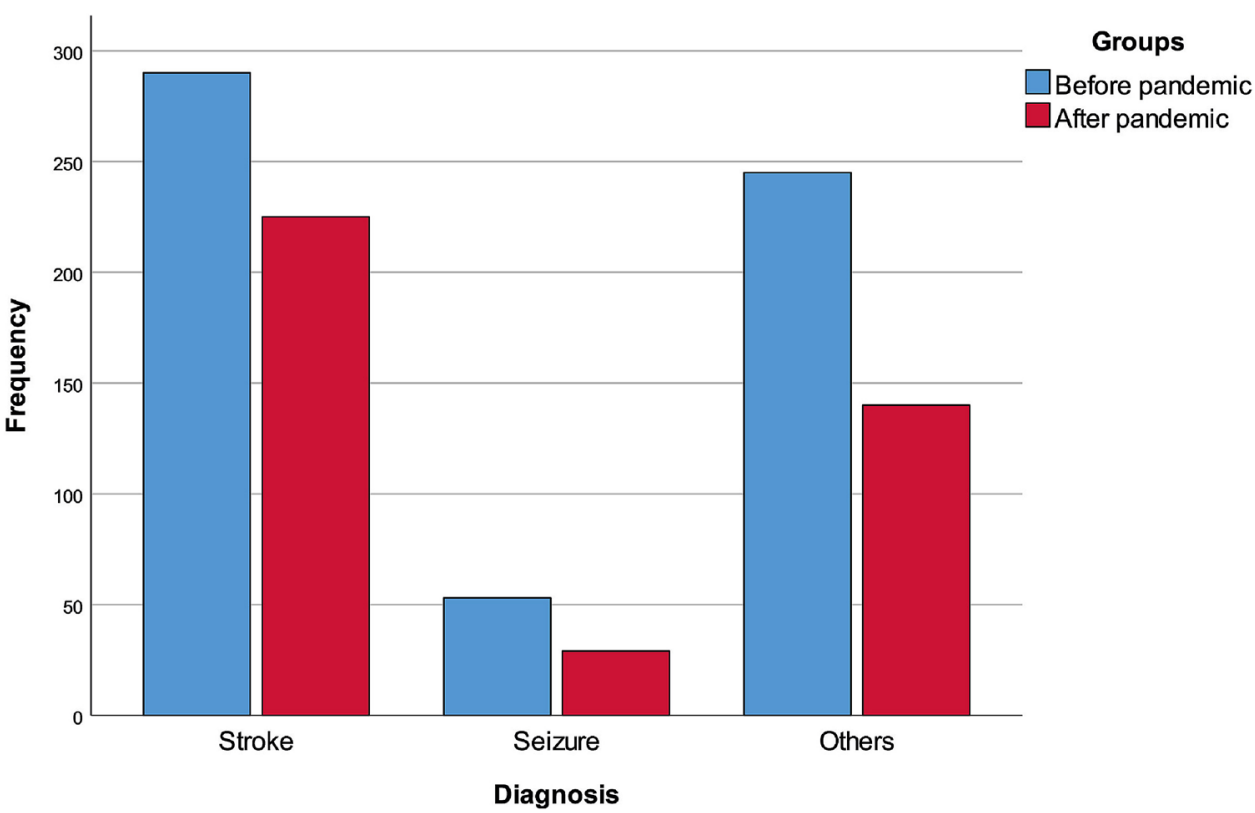


the number of imaging orders for neurology-related cranial $\mathrm{CT}$ and MRI during the pandemic period compared to the pre-pandemic period.

When the literature is reviewed, it can be seen that, during the COVID-19 pandemic period, patient admissions to the emergency department have decreased [16]. In the past, it was observed that the number of patients admitted to the emergency department had also decreased during the SARS epidemic [17]. In the study conducted by Oseran et al., it was reported that there was a $33.7 \%$ decrease in emergency department admissions in 2020 compared to the same dates in 2019 [18]. Again, there are publications reporting that emergency department admissions of life-threatening myocardial infarction, hyperglycemic crisis, surgical emergencies cases and acute orthopedic trauma referrals decreased during the pandemic period [19-23]. In our study, it was found that the number of patients admitted to the emergency department with neurological symptoms decreased by $32.8 \%$ during the pandemic period compared to the prepandemic period.

In the Lombardy region of Italy, where the COVID-19 pandemic had dealt a severe blow, neurologists observed that the number of patients admitted due to stroke decreased during the pandemic period [24]. It has been stated that the fears associated with the pandemic may have caused individuals to overlook mild cerebrovascular disease symptoms. It is also possible that the presence of life-threatening respiratory distress in patients with COVID-19 may have led to reduced awareness of cerebrovascular events developing among these individuals. Again in Italy, a study conducted in a region with a lower prevalence of COVID-19 infection reported that, despite the decrease in the total number of patients admitted to the emergency department during the COVID-19 pandemic, the percentage of requests for neurology consultation did not change and the number of patients with severe neurological conditions requiring hospitalization not decreased [25].

In the literature, there are publications reporting that hospitalizations due to stroke, thrombolytic or thrombectomy treatments decreased during the pandemic COVID-19 period. Rudilosso et al. reported in their study that there were decreases in the number of hospitalizations due to stroke and thrombectomy procedures, and the patients admitted due to stroke were younger compared to the pre-pandemic period [12]. In the study conducted by Uchino et al., a decrease in the number of acute stroke cases and thrombolytic therapy applications were detected in emergency departments during the pandemic [13]. Furthermore, Zhao et al. compared the pandemic period with the same period in 2019 and found that hospital admissions due to stroke decreased by almost half, and the number of thrombolysis and thrombectomy cases decreased by a quarter [14].
In our study, it was detected that the number of patients admitted to the emergency department for stroke decreased by $22.4 \%$ compared to the time period before the pandemic. It was observed that the number of hospitalized patients due to stroke and length of stay decreased during the pandemic period (AP) compared to the pre-pandemic period (BP). The decrease in the number of stroke patients and the duration of hospitalization in the neurology department may be due to a reluctance to remain in the hospital, especially among subjects accompanying patients who were in fear of transmission. It is also possible that, in the absence of definitive findings or due to limited bed capacity, physicians may have elected not to hospitalize suspicious stroke cases with mild neurological symptoms.

Similar to the study by Rudilosso et al. [12], we observed that patients who were diagnosed with stroke during the pandemic period were younger. No statistical difference was observed between the BP and AP groups in terms of ischemic stroke, hemorrhagic stroke and TIA frequency. Again, between the groups, no significant differences were found in terms of the clinical characteristics of stroke, including origin (anterior or posterior system), large or small vessel infarction and mortality.

In a previous publication, it was reported that the number of patients who applied to the emergency department due to seizures during the pandemic period had decreased, and patients with chronic epilepsy applied to the emergency department less frequently during the pandemic. Again, in this study, it was reported that the number of patients admitted with their first seizure was higher, and that examinations such as electroencephalography and brain CT were performed more frequently in patients admitted with seizures during the pandemic period. This situation was explained by the fact that the number of patients applying to the emergency department due to the first seizure was higher; therefore, increasing the use of diagnostic tests in these individuals [26].

In our study, a significant decrease was found in the number of patients admitted with seizures during the pandemic period (AP) compared to the pre-pandemic period (BP) (Table 1). The reason for this may be that patients with chronic epilepsy were more likely to adhere to medication use when in curfew, since they were alone less often and may have received support from their families. When we evaluated patients with seizures, there were no significant differences between the BP and AP groups in terms of age, gender and length of hospital stay. Malignancy history was significantly more common in patients that applied during the pandemic compared to patients that had applied in the previous time period. Unlike the study conducted by Cheli et al. [26], we did not identify a significant difference in the number of patients admitted with their first seizure and the 
number of imaging studies used (CT and MRI) when we compared the pre-pandemic and pandemic periods.

\section{Limitations}

We cannot generalize our results even though our hospital is a very large tertiary healthcare institution which receives referrals at the local and national level. Nevertheless, since it is difficult to ascertain the functionality of referral processes and patient transport during the pandemic, the single-center nature of our study remains as a limitation. In addition, the fact that some data could not be reached due to the retrospective study design is another limitation of our study. It is also possible that the seasonal differences in the time periods was a factor in the number of patients being admitted to the emergency department.

In our study, compared to the pre-pandemic period, it was observed that there was a significant decrease in the number of neurology consultation requests from the emergency department, the number of hospitalizations in the neurology department, and the number of orders for cranial CT and MRI imaging in the pandemic period.

Social isolation measures, curfews and travel restrictions due to the COVID-19 pandemic may have resulted in an avoidance behavior for hospital application among patients (and their relatives) -quite evidently due to the fear of disease contraction. In addition, as mentioned before some relatives of patients requiring hospitalization refused treatment. This may be another factor (in addition to reluctance to apply to hospitals) which reduced the frequency of hospitalization.

\section{Conflict of interest None.}

\section{References}

1. Wu F, Zhao S, Yu B et al (2020) A new coronavirus associated with human respiratory disease in China. Nature 579(7798):265-269

2. Zhu N, Zhang D, Wang W et al (2020) A novel coronavirus from patients with pneumonia in China, 2019. N Engl J Med 382:727-733

3. Coronavirus N. Situation report-10-30 January 2020. Geneva: World Health Organization (2020). https://www.who.int/docs/defau 1t-source/coronaviruse/situation-reports/20200130-sitrep-10-ncov. pdf.2019.

4. Lu R, Zhao X, Li J et al (2020) Genomic characterisation and epidemiology of 2019 novel coronavirus: implications for virus origins and receptor binding. Lancet 395:565-574

5. Zhou P, Yang XL, Wang XG et al (2020) A pneumonia outbreak associated with a new coronavirus of probable bat origin. Nature. https://doi.org/10.1038/s41586-020-2012-7

6. Mao L, Jin H, Wang M et al (2020) Neurologic manifestations of hospitalized patients with coronavirus disease 2019 in Wuhan China. JAMA Neurol 77(6):683-690

7. Liotta EM, Batra A, Clark JR et al (2020) Frequent neurologic manifestations and encephalopathy-associated morbidity in Covid-19 patients. Ann Clin Translat Neurol 7:2221-2230
8. Şeker M, Özer A, Tosun Z, Korkut C, Doğrul M (2020) Covid-19 Pandemi Değerlendirme Raporu. Ankara, Türkiye Bilimler Akademisi Yay

9. Emre U, Demir AS, Acıman E, Çabuk N, Kıran S, Ünal A (2009) The profile of neurology patients evaluated in the emergency department. Turkish J Neurol 15(3):134-139

10. Vural S, Hamamci M, Kilic N (2020) Analysis of the neurology consultations in the emergency department and diagnostic accuracy of emergency physicians for the neurologic emergencies. Ann Med Res 27(6):1797-1802

11. Hartnett K, Kite-Powell A, DeVies J et al (2020) National syndromic surveillance program community of practice. Impact of the COVID-19 pandemic on emergency department visits-United States, January 1, 2019-May 30, 2020. MMWR Morb Mortal Wkly Rep 69(23):699-704

12. Rudilosso S, Laredo C, Vera V et al (2020) Acute stroke care is at risk in the era of COVID-19: experience at a comprehensive stroke center in barcelona. Stroke 51(7):1991-1995

13. Uchino K, Kolikonda MK, Brown D et al (2020) Decline in stroke presentations during COVID-19 surge. Stroke 51(8):2544-2547

14. Zhao J, Li H, Kung D, Fisher M, Shen Y, Liu R (2020) Impact of the COVID-19 epidemic on stroke care and potential solutions. Stroke 51(7):1996-2001

15. Nagamine M, Chow DS, Chang PD, Boden-Albala B, Yu W, Soun JE (2020) Impact of COVID-19 on acute stroke presentation at a comprehensive stroke center. Front Neurol 11:850

16. Boserup B, McKenney M, Elkbuli A (2020) The impact of the COVID-19 pandemic on emergency department visits and patient safety in the United States. Am J Emerg Med 38(9):1732-1736

17. Man CY, Yeung RS, Chung JY, Cameron PA (2003) Impact of SARS on an emergency department in Hong Kong. Emerg Med (Fremantle) 15(5-6):418-422

18. Oseran AS, Nash D, Kim C et al (2020) Changes in hospital admissions for urgent conditions during COVID-19 pandemic. Am J Manag Care 26(8):327-328

19. Garcia S, Albaghdadi MS, Meraj PM et al (2020) Reduction in STSegment elevation cardiac catheterization laboratory activations in the united states during COVID-19 pandemic. J Am Coll Cardiol 75(22):2871-2872

20. Rodriguez-Leor O, Cid-Álvarez B, Ojeda S et al (2020) Impact of the COVID-19 pandemic on interventional cardiology activity in Spain. REC Interv Cardiol 2:82-89

21. Lange SJ, Ritchey MD, Goodman AB et al (2020) Potential Indirect Effects of the COVID-19 pandemic on use of emergency departments for acute life-threatening conditions-United States, januarymay 2020. MMWR Morb Mortal Wkly Rep 69(25):795-800

22. Park C, Sugand K, Nathwani D, Bhattacharya R, Sarraf KM (2020) Impact of the COVID-19 pandemic on orthopedic trauma workload in a London level 1 trauma center: the "golden month." Acta Orthop 91:1-6

23. Göksoy B, Akça MT, Inanç ÖF (2020) The impacts of the COVID19 outbreak on emergency department visits of surgical patients. Turkish J Trauma Emerg Surgery 26(5):685-69224

24. Bersano A, Pantoni L (2020) On being a neurologist in Italy at the time of the COVID-19 outbreak. Neurology 94(21):905-906

25. Caproni S, Di Schino C, Costantini F, Parisi G, Colosimo C (2020) Letter to the editor: "COVID-19 does not reduce stroke-related emergencies.” Acta Neurol Belg 120(5):1259-1260

26. Cheli M, Dinoto A, Olivo S et al (2020) SARS-CoV-2 pandemic and epilepsy: the impact on emergency department attendances for seizures. Seizure 82:23-26

Publisher's Note Springer Nature remains neutral with regard to jurisdictional claims in published maps and institutional affiliations. 